\title{
Are lower limb biomechanical factors associated with night-time calf cramps in adults? A case-control study
}

\author{
Fiona Hawke ${ }^{1,2^{*}}$, Vivienne Chuter ${ }^{1}$, Joshua Burns 2,3 \\ From 4th Congress of the International Foot and Ankle Biomechanics (i-FAB) Community \\ Busan, Korea. 8-11 April 2014
}

\section{Background}

Night-time calf muscle cramps are highly prevalent and are associated with reduced quality of sleep and healthrelated quality of life [1]. The underlying mechanism is poorly understood and no treatment has shown consistent efficacy or safety. The aim of this study was to identify factors associated with night-time calf cramping in adults to explore potential underlying mechanisms and therapeutic targets.

\section{Methods}

160 adults were recruited the Greater Newcastle and Central Coast regions of New South Wales, Australia: 80 who experienced night-time calf cramp at least once per week and 80 age- and sex-matched adults who never experienced lower limb muscle cramping. Participants were assessed using reliable tests of foot/ankle and toe strength, range of ankle dorsiflexion, hamstring flexibility, foot alignment, and calf circumference. Participants also completed a bespoke survey examining health and lifestyle factors, exercise, lower limb symptoms and footwear characteristics.

\section{Results}

Presence of night-time calf cramps was significantly correlated with weakness of foot and ankle inversion, eversion, dorsiflexion and plantarflexion; weakness of toe grip; restricted hamstring flexibility; lower limb tingling sensations; muscle twitching, and coldness of legs or feet in bed at night. Conditional logistic regression identified three factors independently associated with night- time calf cramps: muscle twitching (OR 4.6; 95\%CI: 1.6 to $15.5 ; p=0.01$ ), lower limb tingling (OR 4.1; 95\%CI: 1.6 to $10.3 ; p=0.003$ ) and foot dorsiflexion weakness (OR 1.02; $95 \%$ CI: 1.01 to $1.03 ; p=0.002$ ), which represented other measures of lower limb weakness in the model.

\section{Conclusion}

Night-time calf muscle cramps were associated with markers of neurological dysfunction and potential musculoskeletal therapeutic targets.

\section{Authors' details}

${ }^{1}$ Podiatry Program, The University of Newcastle, Ourimbah, NSW, 2258, Australia. ${ }^{2}$ Sydney Medical School, The University of Sydney, Westmead, NSW, 2145, Australia. ${ }^{3}$ Arthritis and Musculoskeletal Research Group, Faculty of Health Sciences, The University of Sydney / Institute for Neuroscience and Muscle Research / Paediatric Gait Analysis Service of NSW, Sydney Children's Hospitals Network (Randwick and Westmead), Australia.

Published: 8 April 2014

\section{References}

1. Hawke $F$, Chuter $V$, Burns J: Impact of nocturnal calf cramping on quality of sleep and health-related quality of life. Qual Life Res 2013, 22: 1281-1286.

2. Gulich $M$, Heil P, Zeitler H-P: Epidemiology and determinants of nocturnal calf cramps. Eur J Gen Pract 1998, 4:109-13.

doi:10.1186/1757-1146-7-S1-A31

Cite this article as: Hawke et al:: Are lower limb biomechanical factors associated with night-time calf cramps in adults? A case-control study. Journal of Foot and Ankle Research 2014 7(Suppl 1):A31.

\footnotetext{
* Correspondence: Fiona.Hawke@newcastle.edu.au

'Podiatry Program, The University of Newcastle, Ourimbah, NSW, 2258,

Australia

Full list of author information is available at the end of the article
} 